\title{
Proton Transfer and $\mathrm{H}_{2}$ Elimination Reaction of Main Group Hydrides $\mathrm{EH}_{4}^{-}(\mathrm{E}=\mathrm{B}, \mathrm{Al}, \mathrm{Ga})$ with Alcohols
}

Oleg A. Filippov, ${ }^{a}$ Andrey M. Filin, ${ }^{a}$ Viktoria N. Tsupreva, ${ }^{a}$ Natalia V. Belkova, ${ }^{a}$ Agustí Lledós ${ }^{b^{*}}$ Gregori Ujaque, ${ }^{b}$ Lina M. Epstein, ${ }^{a^{*}}$ Elena S. Shubina ${ }^{a^{*}}$

${ }^{a}$ A.N. Nesmeyanov Institute of Organoelement Compounds, Russian Academy of Sciences, 28 Vavilov str., 119991 Moscow, Russia

${ }^{\mathrm{b}}$ Departament de Química, Edifici Cn, Universitat Autònoma de Barcelona, 08193 Bellaterra, Catalonia, Spain

\section{Supporting Information}

\section{DFT/B3LYP optimized structures of dihydrogen bonded complexes (Cartesian coordinates)}

Table S1. $\mathrm{BH}_{4}^{-}$with $\mathrm{HOCH}_{3}$

$\begin{array}{lrrr}\mathrm{O} & 0.935350000 & 0.791309000 & -0.001083000 \\ \mathrm{H} & -0.046568000 & 0.795883000 & 0.019543000 \\ \mathrm{C} & 1.355435000 & -0.557118000 & -0.000166000 \\ \mathrm{H} & 2.442076000 & -0.566939000 & -0.144818000 \\ \mathrm{H} & 1.124972000 & -1.063742000 & 0.947706000 \\ \mathrm{H} & 0.885625000 & -1.134588000 & -0.806329000 \\ \mathrm{~B} & -2.225275000 & -0.119552000 & -0.000930000 \\ \mathrm{H} & -1.687110000 & 1.000890000 & -0.007892000 \\ \mathrm{H} & -1.955783000 & -0.708146000 & -1.049017000 \\ \mathrm{H} & -3.438966000 & 0.053751000 & 0.092328000 \\ \mathrm{H} & -1.813281000 & -0.767109000 & 0.962794000\end{array}$

Table S2. $\mathrm{BH}_{4}{ }^{-}$with $\mathrm{CF}_{3} \mathrm{CH}_{2} \mathrm{OH}$

$\begin{array}{lrrr}\mathrm{O} & 0.993252000 & 1.181809000 & -0.001815000 \\ \mathrm{H} & 1.931823000 & 0.933017000 & 0.188066000 \\ \mathrm{C} & 0.377255000 & 0.062148000 & -0.566921000 \\ \mathrm{H} & 0.254194000 & 0.159650000 & -1.655649000 \\ \mathrm{H} & 0.938581000 & -0.855652000 & -0.366193000 \\ \mathrm{C} & -1.017019000 & -0.112380000 & 0.001792000 \\ \mathrm{~F} & -1.820408000 & 0.960459000 & -0.219214000\end{array}$




$\begin{array}{lrrr}\text { F } & -1.038027000 & -0.340660000 & 1.331616000 \\ \text { F } & -1.639385000 & -1.177997000 & -0.586788000 \\ \text { B } & 3.693694000 & -0.492013000 & 0.052049000 \\ \text { H } & 3.001113000 & -1.369883000 & 0.566954000 \\ \text { H } & 3.406359000 & 0.611105000 & 0.553626000 \\ \text { H } & 4.878605000 & -0.692610000 & 0.288740000 \\ \text { H } & 3.493809000 & -0.454860000 & -1.161026000\end{array}$

Table S3 $\mathrm{BH}_{4}{ }^{-}$with $\mathrm{CF}_{3} \mathrm{OH}$

$\begin{array}{lrrr}\mathrm{O} & 0.362764000 & 0.836975000 & 0.001325000 \\ \mathrm{H} & 1.270146000 & 0.331390000 & -0.000601000 \\ \mathrm{C} & -0.669843000 & 0.048696000 & 0.000006000 \\ \mathrm{~B} & 3.327909000 & -0.009279000 & 0.000196000 \\ \mathrm{H} & 2.233018000 & -0.616016000 & -0.002989000 \\ \mathrm{H} & 3.915264000 & -0.361009000 & -1.013763000 \\ \mathrm{H} & 3.910250000 & -0.361100000 & 1.016987000 \\ \mathrm{H} & 3.147604000 & 1.201565000 & -0.000244000 \\ \mathrm{~F} & -1.823476000 & 0.776490000 & -0.001481000 \\ \mathrm{~F} & -0.756347000 & -0.784548000 & 1.086990000 \\ \mathrm{~F} & -0.753387000 & -0.784877000 & -1.086733000\end{array}$

Table $44 \mathrm{AlH}_{4}^{-}$with $\mathrm{CH}_{3} \mathrm{OH}$

$\begin{array}{lrrr}\mathrm{O} & 1.852175000 & -0.787639000 & -0.005858000 \\ \mathrm{H} & 0.879400000 & -0.898857000 & 0.070286000 \\ \mathrm{C} & 2.115936000 & 0.601754000 & -0.000604000 \\ \mathrm{H} & 3.182564000 & 0.737835000 & -0.206603000 \\ \mathrm{H} & 1.538725000 & 1.135641000 & -0.765949000 \\ \mathrm{H} & 1.892799000 & 1.064850000 & 0.971757000 \\ \mathrm{Al} & -2.065879000 & 0.042207000 & -0.002167000 \\ \mathrm{H} & -0.734668000 & -0.916390000 & 0.230201000 \\ \mathrm{H} & -1.616287000 & 1.316429000 & -0.927093000 \\ \mathrm{H} & -2.588787000 & 0.551773000 & 1.461837000 \\ \mathrm{H} & -3.210335000 & -0.849390000 & -0.755771000\end{array}$

Table S5 $\mathrm{AlH}_{4}^{-}$with $\mathrm{CF}_{3} \mathrm{CH}_{2} \mathrm{OH}$

$\begin{array}{lrrr}\mathrm{O} & -0.309872000 & 0.865324000 & -0.002864000 \\ \mathrm{H} & 0.616741000 & 0.372280000 & 0.005082000 \\ \mathrm{C} & -1.323694000 & 0.049461000 & 0.000029000 \\ \mathrm{Al} & 3.377860000 & -0.005179000 & -0.000355000 \\ \mathrm{H} & 1.718566000 & -0.234797000 & 0.019637000 \\ \mathrm{H} & 3.945002000 & -1.011265000 & -1.144336000 \\ \mathrm{H} & 3.901124000 & -0.396771000 & 1.488020000 \\ \mathrm{H} & 3.637957000 & 1.557637000 & -0.360557000 \\ \mathrm{~F} & -2.495540000 & 0.743406000 & -0.009698000 \\ \mathrm{~F} & -1.384545000 & -0.776500000 & 1.092746000 \\ \mathrm{~F} & -1.376630000 & -0.793473000 & -1.080880000\end{array}$

Table S6. $\mathrm{AlH}_{4}{ }^{-}$with $\mathrm{CF}_{3} \mathrm{OH}$

$\begin{array}{rrrr}\mathrm{O} & 0.215702000 & 1.498607000 & 0.062611000 \\ \mathrm{H} & 1.134254000 & 1.261787000 & 0.349879000 \\ \mathrm{C} & -0.291034000 & 0.421959000 & -0.672327000\end{array}$




$\begin{array}{lrrr}\mathrm{H} & -0.615452000 & 0.745287000 & -1.670277000 \\ \mathrm{H} & 0.443957000 & -0.381858000 & -0.792832000 \\ \mathrm{C} & -1.519270000 & -0.172238000 & -0.003963000 \\ \mathrm{~F} & -2.520750000 & 0.730641000 & 0.152412000 \\ \mathrm{~F} & -1.271151000 & -0.691006000 & 1.215392000 \\ \mathrm{~F} & -2.032975000 & -1.181803000 & -0.766332000 \\ \mathrm{Al} & 3.574335000 & -0.290657000 & 0.012123000 \\ \mathrm{H} & 2.690219000 & -1.175842000 & -1.045555000 \\ \mathrm{H} & 2.519006000 & 0.779768000 & 0.722268000 \\ \mathrm{H} & 4.201809000 & -1.229194000 & 1.185745000 \\ \mathrm{H} & 4.719949000 & 0.570924000 & -0.763229000\end{array}$

Table S7 $\mathrm{GaH}_{4}^{-}$with $\mathrm{CH}_{3} \mathrm{OH}$

$\begin{array}{lrrr}\mathrm{O} & -2.521624000 & -0.787993000 & -0.009657000 \\ \mathrm{H} & -1.552939000 & -0.902448000 & 0.103324000 \\ \mathrm{C} & -2.782543000 & 0.602402000 & -0.000012000 \\ \mathrm{H} & -2.664441000 & 1.040964000 & 1.002089000 \\ \mathrm{H} & -2.123949000 & 1.149717000 & -0.684946000 \\ \mathrm{Ga} & 1.347064000 & 0.020852000 & -0.001697000 \\ \mathrm{H} & 1.987315000 & 0.502008000 & 1.399328000 \\ \mathrm{H} & 0.058156000 & -0.932602000 & 0.335756000 \\ \mathrm{H} & 0.824518000 & 1.289441000 & -0.855219000 \\ \mathrm{H} & 2.401349000 & -0.851714000 & -0.854314000 \\ \mathrm{H} & -3.820739000 & 0.747744000 & -0.316098000\end{array}$

Table S8. $\mathrm{GaH}_{4}^{-}$with $\mathrm{CF}_{3} \mathrm{CH}_{2} \mathrm{OH}$

$\begin{array}{lrrr}\mathrm{O} & -0.566020000 & 1.516910000 & 0.051918000 \\ \mathrm{H} & 0.356330000 & 1.309271000 & 0.348329000 \\ \mathrm{C} & -1.040692000 & 0.411537000 & -0.661059000 \\ \mathrm{H} & -1.343826000 & 0.697253000 & -1.677169000 \\ \mathrm{H} & -0.292914000 & -0.385722000 & -0.735950000 \\ \mathrm{C} & -2.277939000 & -0.175779000 & -0.003173000 \\ \mathrm{~F} & -3.292082000 & 0.720150000 & 0.103968000 \\ \mathrm{~F} & -2.052735000 & -0.655974000 & 1.236599000 \\ \mathrm{~F} & -2.764224000 & -1.213101000 & -0.746186000 \\ \mathrm{Ga} & 2.824851000 & -0.141113000 & 0.006084000 \\ \mathrm{H} & 1.960489000 & -1.134782000 & -0.931311000 \\ \mathrm{H} & 1.758356000 & 0.874025000 & 0.742080000 \\ \mathrm{H} & 3.589063000 & -0.952332000 & 1.162301000 \\ \mathrm{H} & 3.823437000 & 0.757283000 & -0.876264000\end{array}$

Table S9. $\mathrm{GaH}_{4}{ }^{-}$with $\mathrm{CF}_{3} \mathrm{OH}$

$\begin{array}{lrrr}\mathrm{O} & -1.076788000 & 0.851802000 & 0.004573000 \\ \mathrm{H} & -0.157553000 & 0.346623000 & 0.014578000 \\ \mathrm{C} & -2.100836000 & 0.048750000 & 0.000087000 \\ \mathrm{~F} & -2.159960000 & -0.790247000 & -1.082950000 \\ \mathrm{~F} & -2.177615000 & -0.779616000 & 1.090518000 \\ \mathrm{Ga} & 2.575238000 & -0.001699000 & -0.000382000 \\ \mathrm{H} & 3.122192000 & -0.798882000 & -1.279447000 \\ \mathrm{H} & 0.936586000 & -0.296422000 & 0.029501000 \\ \mathrm{H} & 3.134404000 & -0.585135000 & 1.384714000\end{array}$




$\begin{array}{lrrr}\mathrm{H} & 2.760759000 & 1.584800000 & -0.128096000 \\ \mathrm{~F} & -3.263475000 & 0.758168000 & -0.012737000\end{array}$

DFT/B3LYP optimized structures of transition states

Table S10. TS for $\mathrm{BH}_{4}{ }^{-}$with $\mathrm{CH}_{3} \mathrm{OH}$

$\begin{array}{lrrr}\mathrm{O} & -0.531476000 & -0.604077000 & -0.342230000 \\ \mathrm{H} & 0.439282000 & -0.952003000 & 0.794523000 \\ \mathrm{C} & -1.389646000 & 0.354272000 & 0.109787000 \\ \mathrm{H} & -1.358539000 & 1.304654000 & -0.478895000 \\ \mathrm{H} & -2.463937000 & 0.041653000 & 0.098651000 \\ \mathrm{H} & -1.190504000 & 0.667088000 & 1.170373000 \\ \mathrm{~B} & 1.978939000 & 0.303947000 & -0.096835000 \\ \mathrm{H} & 1.011376000 & -1.038925000 & 1.393694000 \\ \mathrm{H} & 1.893069000 & -0.014505000 & -1.241736000 \\ \mathrm{H} & 2.883814000 & -0.144093000 & 0.550484000 \\ \mathrm{H} & 1.480427000 & 1.323381000 & 0.276197000\end{array}$

Table S11. TS for $\mathrm{BH}_{4}{ }^{-}$with $\mathrm{CF}_{3} \mathrm{CH}_{2} \mathrm{OH}$

$\begin{array}{lrrr}\mathrm{O} & 1.224143000 & -0.532672000 & 0.433710000 \\ \mathrm{H} & 2.304781000 & -0.995975000 & -0.822511000 \\ \mathrm{C} & 0.415019000 & 0.412240000 & -0.062185000 \\ \mathrm{H} & 0.447046000 & 1.398309000 & 0.468177000 \\ \mathrm{H} & 0.584788000 & 0.662820000 & -1.145049000 \\ \mathrm{C} & -1.076478000 & 0.049606000 & -0.022006000 \\ \mathrm{~F} & -1.556545000 & -0.156473000 & 1.233646000 \\ \mathrm{~F} & -1.391040000 & -1.066655000 & -0.730497000 \\ \mathrm{~F} & -1.865503000 & 1.052466000 & -0.551633000 \\ \mathrm{~B} & 3.882951000 & 0.357297000 & 0.035933000 \\ \mathrm{H} & 3.342018000 & 1.349012000 & -0.346038000 \\ \mathrm{H} & 2.845279000 & -1.094925000 & -1.393707000 \\ \mathrm{H} & 4.744023000 & -0.117068000 & -0.647099000 \\ \mathrm{H} & 3.810723000 & 0.037588000 & 1.178396000\end{array}$

Table S12. TS1 for $\mathrm{BH}_{4}{ }^{-}$with $\mathrm{CF}_{3} \mathrm{OH}$

$\begin{array}{cccc}\text { H } & -1.717025000 & -0.067721000 & -0.048087000 \\ \text { B } & -3.341864000 & -0.032541000 & 0.015273000 \\ \text { H } & -2.235403000 & 0.650928000 & -0.140158000 \\ \text { H } & -3.907545000 & 0.363154000 & -0.977576000 \\ \text { H } & -3.739119000 & 0.433323000 & 1.056371000 \\ \text { H } & -3.182943000 & -1.223403000 & 0.037218000 \\ \text { C } & 0.635282000 & -0.085667000 & -0.000668000 \\ \text { F } & 0.958039000 & 0.553277000 & 1.216621000 \\ \text { F } & 0.692625000 & 0.984402000 & -0.922581000 \\ \text { F } & 1.797230000 & -0.813199000 & -0.287659000 \\ \text { O } & -0.418923000 & -0.749987000 & -0.007194000\end{array}$


Table S13. TS2 for $\mathrm{BH}_{4}{ }^{-}$with $\mathrm{CF}_{3} \mathrm{OH}$

$\begin{array}{cccc}\mathrm{H} & -1.983976000 & -1.714305000 & -0.091446000 \\ \mathrm{~B} & -3.521962000 & 0.359256000 & -0.001207000 \\ \mathrm{H} & -2.634643000 & -2.067267000 & 0.059383000 \\ \mathrm{H} & -2.770748000 & 1.176555000 & 0.415778000 \\ \mathrm{H} & -4.212986000 & -0.256456000 & 0.753737000 \\ \mathrm{H} & -3.702692000 & 0.248078000 & -1.172929000 \\ \mathrm{C} & 0.642945000 & -0.035439000 & -0.082820000 \\ \mathrm{~F} & 1.859458000 & -0.654126000 & -0.524499000 \\ \mathrm{~F} & 0.736256000 & -0.225391000 & 1.337270000 \\ \mathrm{~F} & 0.978428000 & 1.351641000 & -0.199948000 \\ \mathrm{O} & -0.388762000 & -0.402421000 & -0.622123000\end{array}$

Table S14. TS for $\mathrm{AlH}_{4}{ }^{-}$with $\mathrm{CH}_{3} \mathrm{OH}$

$\begin{array}{lrrc}\mathrm{O} & 0.763808000 & -0.565236000 & -0.272207000 \\ \mathrm{H} & 0.215654000 & -1.066305000 & 0.652425000 \\ \mathrm{C} & 1.834298000 & 0.320289000 & 0.087126000 \\ \mathrm{H} & 1.743284000 & 1.258281000 & -0.466852000 \\ \mathrm{H} & 1.817198000 & 0.569447000 & 1.160453000 \\ \mathrm{H} & 2.807892000 & -0.130748000 & -0.142567000 \\ \mathrm{Al} & -1.367105000 & 0.141350000 & -0.023900000 \\ \mathrm{H} & -0.600539000 & -1.191980000 & 1.303238000 \\ \mathrm{H} & -1.898599000 & -0.650319000 & -1.337608000 \\ \mathrm{H} & -0.800135000 & 1.667325000 & -0.229075000 \\ \mathrm{H} & -2.628635000 & 0.306908000 & 1.025576000\end{array}$

Table S15. TS for $\mathrm{AlH}_{4}^{-}$with $\mathrm{CF}_{3} \mathrm{CH}_{2} \mathrm{OH}$

$\begin{array}{lrrr}\mathrm{O} & 0.846656000 & -0.483941000 & 0.248730000 \\ \mathrm{H} & 1.632121000 & -0.719269000 & -0.856774000 \\ \mathrm{C} & -0.045155000 & 0.528979000 & 0.030133000 \\ \mathrm{H} & -0.020640000 & 1.308692000 & 0.812377000 \\ \mathrm{H} & 0.101613000 & 1.057363000 & -0.935423000 \\ \mathrm{C} & -1.488026000 & 0.039481000 & -0.008278000 \\ \mathrm{~F} & -1.897435000 & -0.534632000 & 1.148442000 \\ \mathrm{~F} & -1.724771000 & -0.865121000 & -0.990881000 \\ \mathrm{~F} & -2.356913000 & 1.079162000 & -0.238249000 \\ \mathrm{Al} & 3.193871000 & 0.116987000 & 0.033729000 \\ \mathrm{H} & 2.700061000 & 1.637319000 & 0.264150000 \\ \mathrm{H} & 2.315173000 & -0.738932000 & -1.431087000 \\ \mathrm{H} & 4.496653000 & 0.145783000 & -0.955111000 \\ \mathrm{H} & 3.492604000 & -0.865685000 & 1.268611000\end{array}$

Table S16. TS for $\mathrm{AlH}_{4}{ }^{-}$with $\mathrm{CF}_{3} \mathrm{OH}$

$\begin{array}{lrrr}\mathrm{O} & 0.037461000 & -0.157522000 & -0.575134000 \\ \mathrm{H} & 1.311719000 & -1.304460000 & -0.144165000 \\ \mathrm{C} & -1.099946000 & -0.003544000 & -0.072956000 \\ \mathrm{Al} & 2.994278000 & 0.126060000 & 0.000037000 \\ \mathrm{H} & 1.984419000 & -1.665606000 & 0.009297000 \\ \mathrm{H} & 4.226106000 & -0.864763000 & 0.333852000 \\ \mathrm{H} & 2.488115000 & 0.980132000 & 1.242424000 \\ \mathrm{H} & 2.981193000 & 0.656736000 & -1.501332000\end{array}$




$\begin{array}{lrrr}\text { F } & -1.493089000 & 1.361512000 & 0.245005000 \\ \text { F } & -1.343595000 & -0.694670000 & 1.198710000 \\ \text { F } & -2.231891000 & -0.462328000 & -0.877243000\end{array}$

Table S17. TS for $\mathrm{GaH}_{4}{ }^{-}$with $\mathrm{CH}_{3} \mathrm{OH}$

$\begin{array}{lrrr}\mathrm{O} & -1.380600000 & -0.594426000 & -0.299389000 \\ \mathrm{H} & -0.698210000 & -1.034452000 & 0.748141000 \\ \mathrm{C} & -2.315835000 & 0.350445000 & 0.094716000 \\ \mathrm{H} & -2.239394000 & 0.596983000 & 1.177483000 \\ \mathrm{H} & -2.199636000 & 1.306897000 & -0.446842000 \\ \mathrm{Ga} & 0.955033000 & 0.070076000 & -0.018987000 \\ \mathrm{H} & 0.434773000 & 1.548854000 & -0.328855000 \\ \mathrm{H} & -0.053468000 & -1.166637000 & 1.392076000 \\ \mathrm{H} & 1.383657000 & -0.889834000 & -1.209484000 \\ \mathrm{H} & 2.060430000 & 0.113025000 & 1.158166000 \\ \mathrm{H} & -3.354367000 & 0.005563000 & -0.075261000\end{array}$

Table S18. TS for $\mathrm{GaH}_{4}{ }^{-}$with $\mathrm{CF}_{3} \mathrm{CH}_{2} \mathrm{OH}$

$\begin{array}{lrrr}\mathrm{O} & 0.086704000 & -0.539486000 & 0.313493000 \\ \mathrm{H} & 0.968504000 & -0.939203000 & -0.851295000 \\ \mathrm{C} & -0.757053000 & 0.462190000 & -0.033864000 \\ \mathrm{H} & -0.711684000 & 1.348958000 & 0.633412000 \\ \mathrm{H} & -0.603000000 & 0.857835000 & -1.066500000 \\ \mathrm{C} & -2.227914000 & 0.047728000 & -0.013159000 \\ \mathrm{~F} & -2.664556000 & -0.352103000 & 1.207529000 \\ \mathrm{~F} & -2.519944000 & -0.962956000 & -0.871148000 \\ \mathrm{~F} & -3.050524000 & 1.091258000 & -0.376296000 \\ \mathrm{Ga} & 2.624809000 & 0.076486000 & 0.016978000 \\ \mathrm{H} & 2.088579000 & 1.565979000 & 0.053547000 \\ \mathrm{H} & 1.583863000 & -1.031362000 & -1.413256000 \\ \mathrm{H} & 3.791375000 & -0.123939000 & -1.072235000 \\ \mathrm{H} & 2.844665000 & -0.778734000 & 1.323444000\end{array}$

Table S19. TS for $\mathrm{GaH}_{4}{ }^{-}$with $\mathrm{CF}_{3} \mathrm{OH}$

$\begin{array}{lrrr}\mathrm{O} & -0.670731000 & -0.246130000 & -0.596311000 \\ \mathrm{H} & 0.614181000 & -1.506099000 & -0.138451000 \\ \mathrm{C} & -1.783121000 & -0.014141000 & -0.077841000 \\ \mathrm{~F} & -2.962621000 & -0.412153000 & -0.852421000 \\ \mathrm{~F} & -2.051310000 & -0.666312000 & 1.212889000 \\ \mathrm{Ga} & 2.280969000 & 0.071043000 & 0.000249000 \\ \mathrm{H} & 3.462510000 & -0.934395000 & 0.355779000 \\ \mathrm{H} & 1.272871000 & -1.870928000 & 0.016139000 \\ \mathrm{H} & 1.704648000 & 0.892323000 & 1.197149000 \\ \mathrm{H} & 2.228438000 & 0.543253000 & -1.490401000 \\ \mathrm{~F} & -2.089193000 & 1.381508000 & 0.227269000\end{array}$

Table S20. DFT/B3LYP optimized structure of the $\eta^{2}-\mathrm{H}_{2}$ intermediate for the reaction of $\mathrm{BH}_{4}{ }^{-}$with $\mathrm{CF}_{3} \mathrm{OH}$

$\begin{array}{llll}\mathrm{H} & -1.949753000 & -0.103038000 & -0.009899000 \\ \mathrm{~B} & -3.413230000 & -0.036131000 & 0.004391000\end{array}$




$\begin{array}{cccc}\text { H } & -2.281372000 & 0.666573000 & -0.060486000 \\ \text { H } & -3.879378000 & 0.333553000 & -1.041627000 \\ \text { H } & -3.851552000 & 0.472242000 & 1.003074000 \\ \text { H } & -3.270500000 & -1.225029000 & 0.083278000 \\ \text { C } & 0.649302000 & -0.107571000 & 0.000179000 \\ \text { F } & 1.457182000 & -0.141239000 & 1.165958000 \\ \text { F } & 0.408870000 & 1.298514000 & -0.144517000 \\ \text { F } & 1.601022000 & -0.347670000 & -1.023059000 \\ \text { O } & -0.350097000 & -0.825583000 & 0.002148000\end{array}$

\section{Optimized structures of dihydrogen bonded complexes in THF}

Table S21. $\mathrm{AlH}_{4}^{-}$with $\mathrm{CF}_{3} \mathrm{OH}$

$\begin{array}{lccc}\mathrm{O} & 0.307933000 & 0.863851000 & -0.038724000 \\ \mathrm{H} & -0.581368000 & 0.361098000 & -0.073319000 \\ \mathrm{C} & 1.333689000 & 0.043412000 & -0.001017000 \\ \mathrm{Al} & -3.368020000 & -0.004635000 & 0.002315000 \\ \mathrm{H} & -1.745045000 & -0.315009000 & -0.106174000 \\ \mathrm{H} & -4.117708000 & -1.257048000 & -0.708533000 \\ \mathrm{H} & -3.657510000 & 1.390537000 & -0.775804000 \\ \mathrm{H} & -3.732667000 & 0.102534000 & 1.581304000 \\ \mathrm{~F} & 1.429802000 & -0.767193000 & -1.096701000 \\ \mathrm{~F} & 1.324097000 & -0.799973000 & 1.072554000 \\ \mathrm{~F} & 2.485318000 & 0.745707000 & 0.065073000\end{array}$

Table S22. $\mathrm{GaH}_{4}^{-}$with $\mathrm{CF}_{3} \mathrm{OH}$

$\begin{array}{llcc}\mathrm{O} & 1.051627000 & 0.847701000 & 0.025640000 \\ \mathrm{H} & 0.174036000 & 0.329004000 & 0.061848000 \\ \mathrm{C} & 2.090899000 & 0.043157000 & 0.000561000 \\ \mathrm{~F} & 2.189650000 & -0.761325000 & 1.099778000 \\ \mathrm{~F} & 2.102486000 & -0.805983000 & -1.068529000 \\ \mathrm{Ga} & -2.545460000 & -0.001149000 & -0.001287000 \\ \mathrm{H} & -3.242535000 & -0.467242000 & 1.362740000 \\ \mathrm{H} & -0.963201000 & -0.424807000 & 0.129930000 \\ \mathrm{H} & -3.116987000 & -0.789277000 & -1.271917000 \\ \mathrm{H} & -2.612086000 & 1.585781000 & -0.197979000 \\ \mathrm{~F} & 3.231376000 & 0.763043000 & -0.059382000\end{array}$


Table S23. Dihydrogen bonded complex of $\mathrm{BH}_{4}{ }^{-}$with $\mathrm{CH}_{3} \mathrm{OH}$

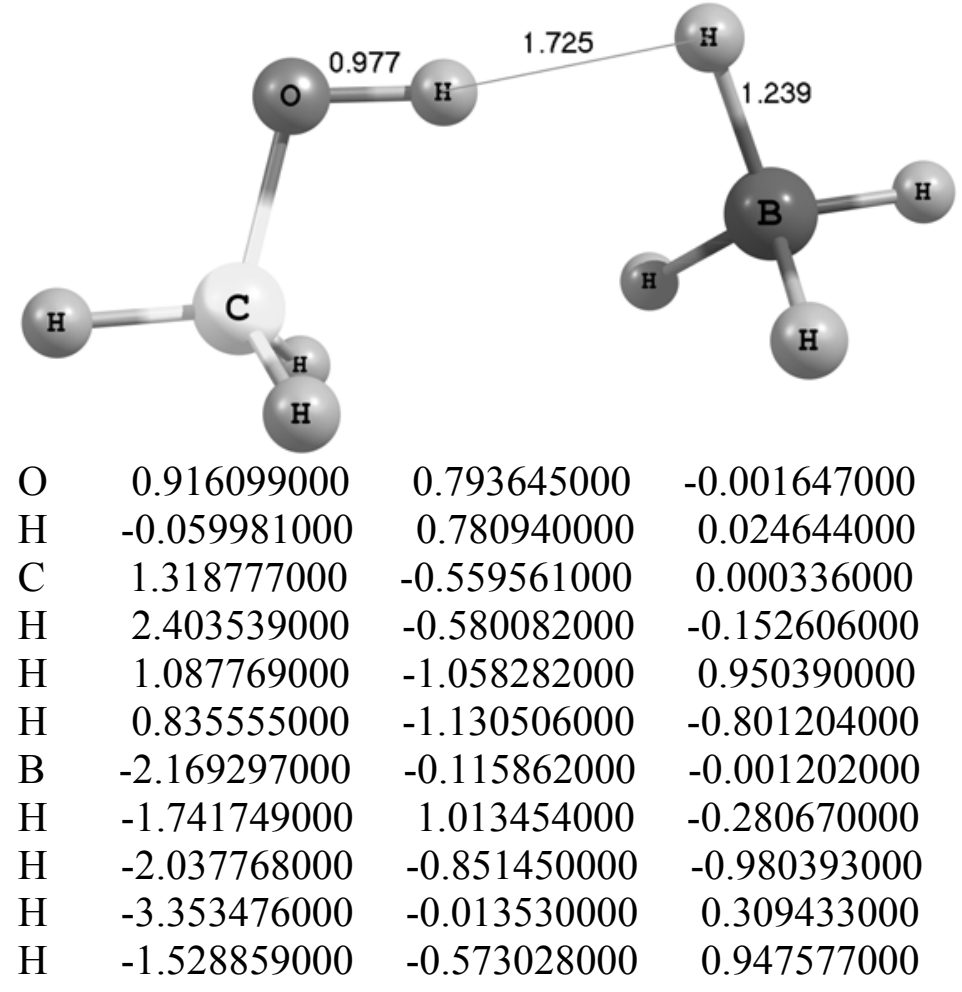

Table S24. Dihydrogen bonded complex of $\mathrm{GaH}_{4}{ }^{-}$with $\mathrm{CH}_{3} \mathrm{OH}$

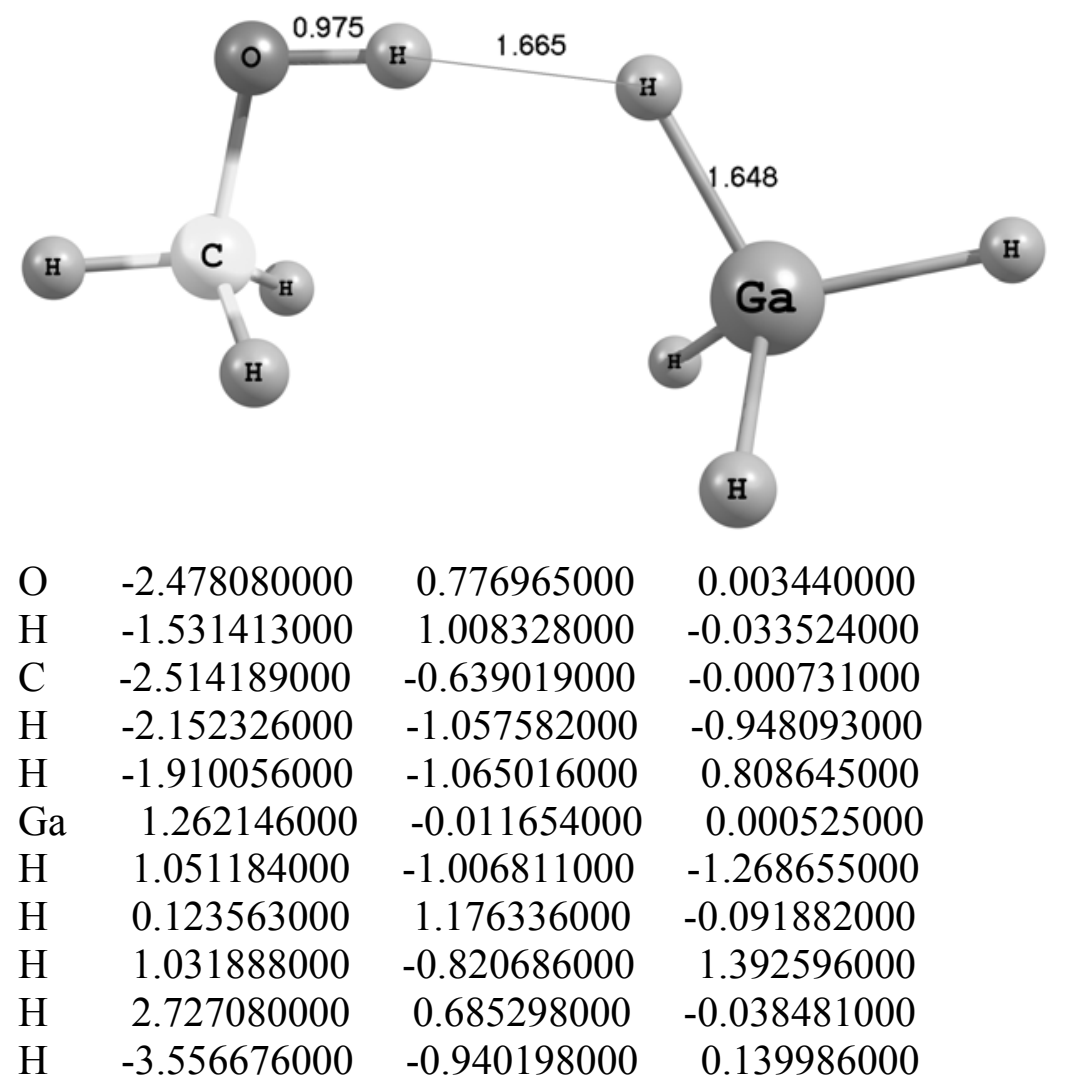


Table S25. Dihydrogen bonded complex of $\mathrm{BH}_{4}{ }^{-}$with $\mathrm{CF}_{3} \mathrm{OH}$

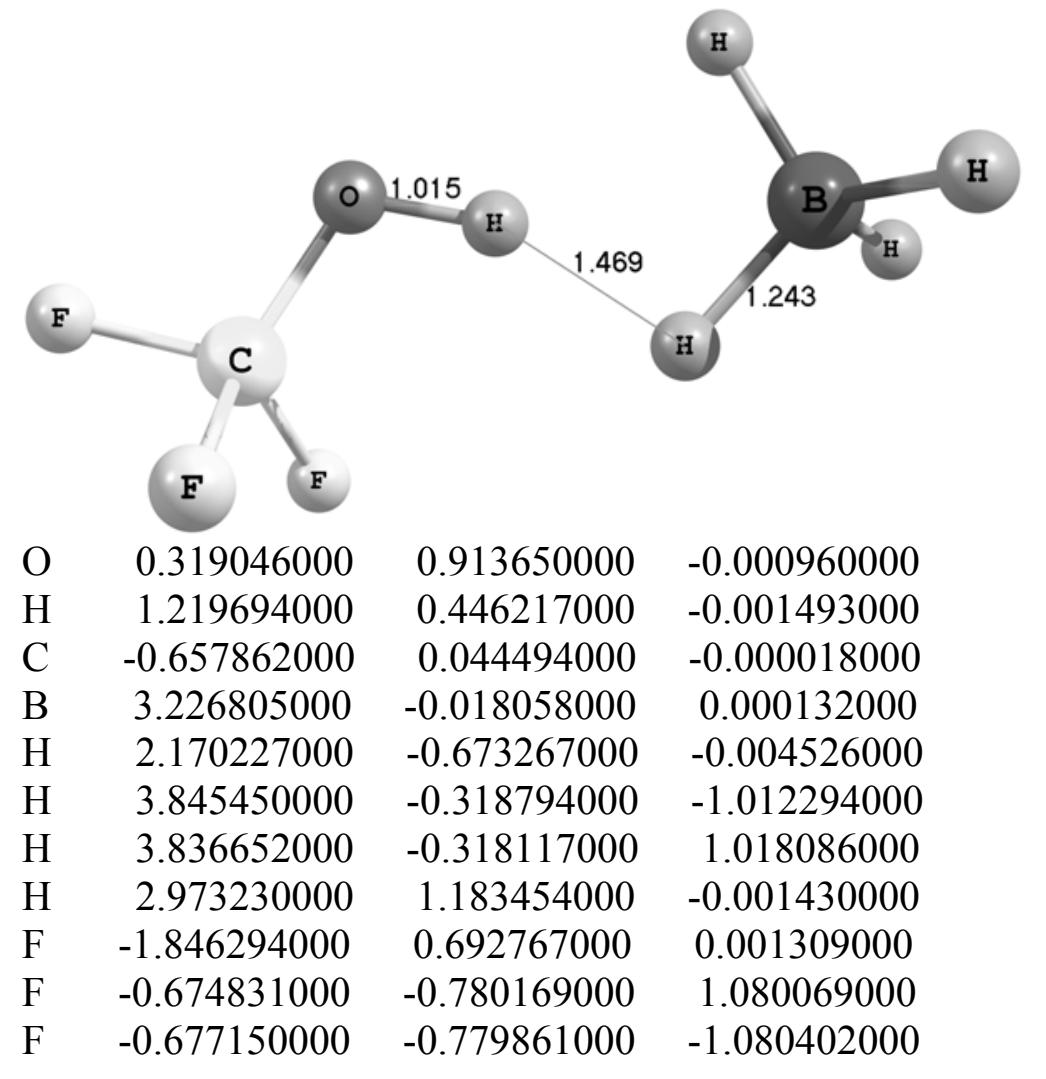

Table S26. TS for $\mathrm{BH}_{4}{ }^{-}$with $\mathrm{CH}_{3} \mathrm{OH}$

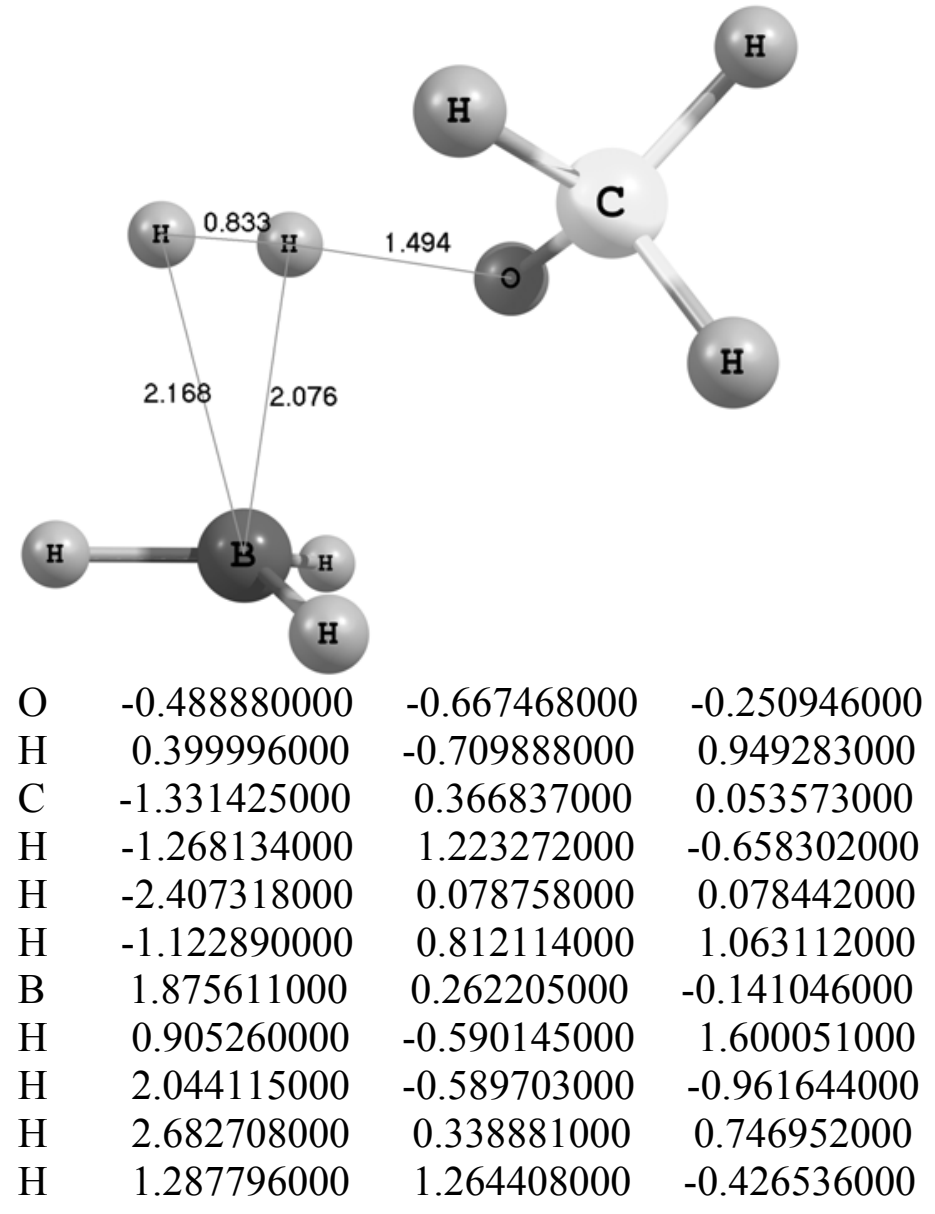


Table S27. TS1 for $\mathrm{BH}_{4}^{-}$with $\mathrm{CF}_{3} \mathrm{OH}$

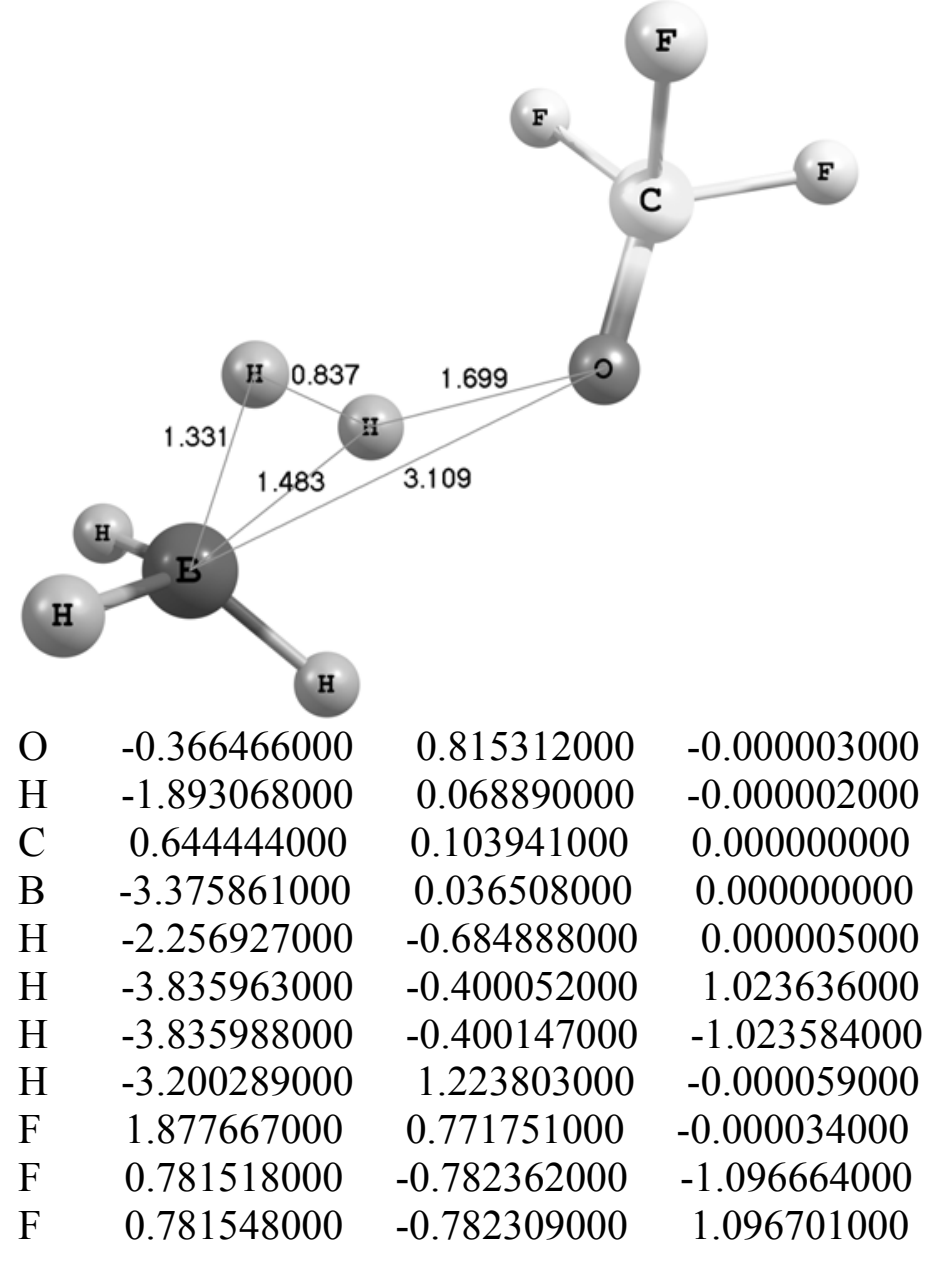

Table S28. $\eta^{2}-\mathrm{H}_{2}$ intermediate of $\mathrm{BH}_{4}{ }^{-}$with $\mathrm{CF}_{3} \mathrm{OH}$

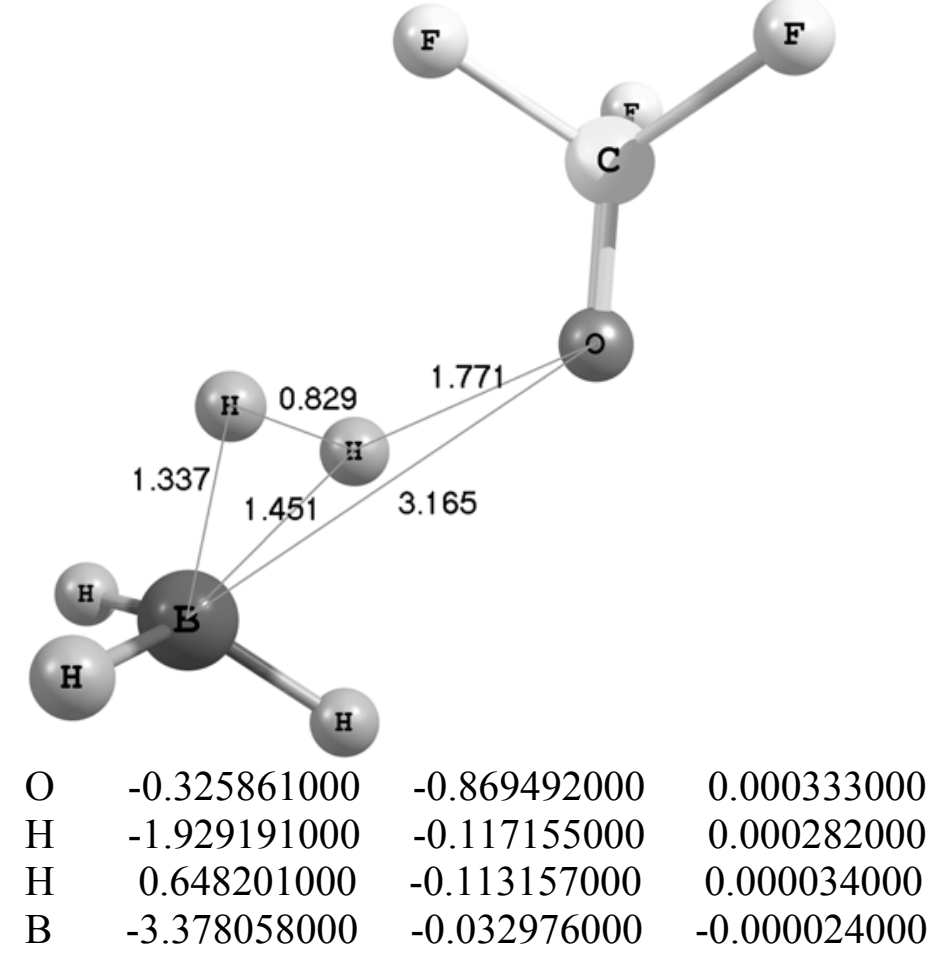




$\begin{array}{cccc} & & & \\ \mathrm{H} & -2.231490000 & 0.654377000 & 0.000102000 \\ \mathrm{H} & -3.818473000 & 0.419131000 & -1.024465000 \\ \mathrm{H} & -3.819042000 & 0.420291000 & 1.023664000 \\ \mathrm{H} & -3.249070000 & -1.226715000 & 0.000718000 \\ \mathrm{~F} & 1.528669000 & -0.214836000 & 1.095740000 \\ \mathrm{~F} & 0.347849000 & 1.281753000 & -0.001412000 \\ \mathrm{~F} & 1.529621000 & -0.216936000 & -1.094667000\end{array}$

Table S29. $\mathrm{TS} 2$ for $\mathrm{BH}_{4}{ }^{-}$with $\mathrm{CF}_{3} \mathrm{OH}$

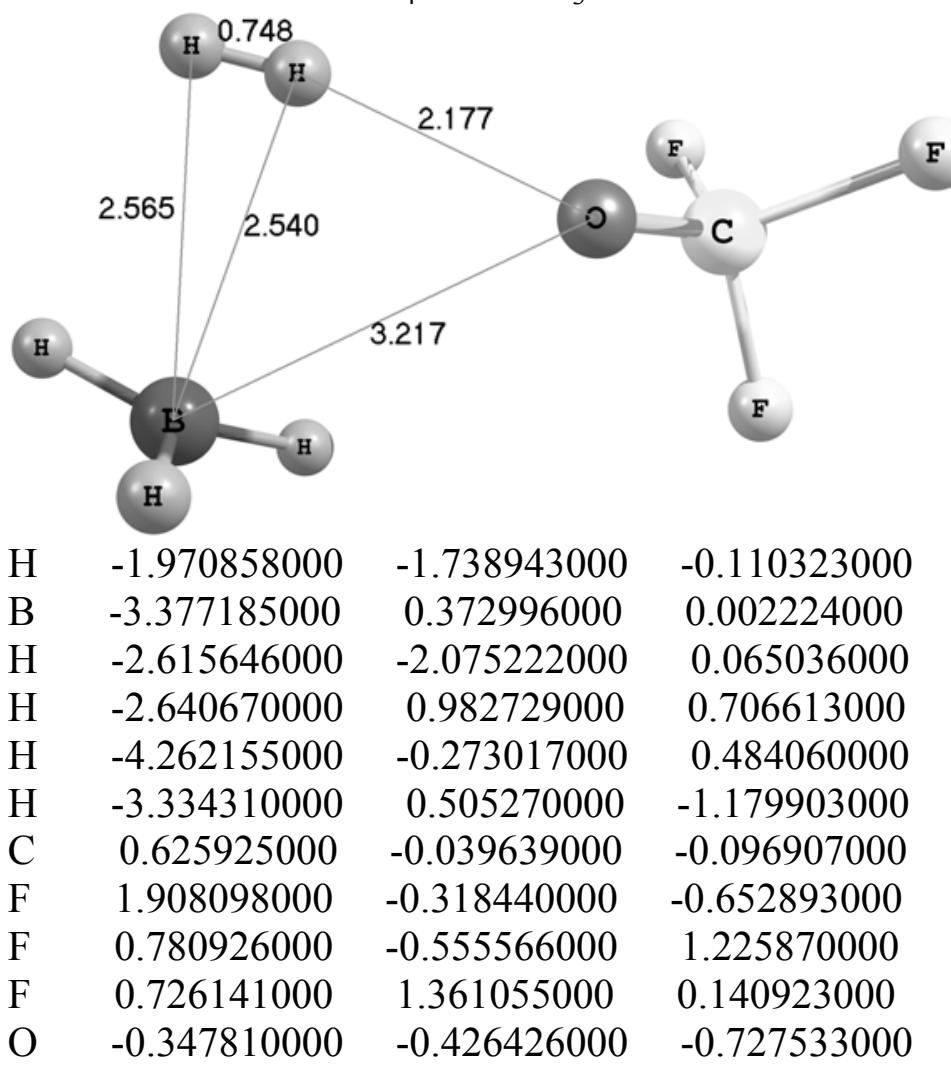

Si 